\section{AB007. Modalities of tracheal resection and reconstruction- the Shanghai Chest Hospital experience}

\author{
Heng Zhao, Feng Yao, Yang Zhao, Hai Yong Gu \\ Department of Thoracic Surgery, Shanghai Chest Hospital, Shanghai \\ Jiaotong University, Shanghai 200030, China
}

Correspondence to: Heng Zhao, MD, PhD. Shanghai Chest Hospital, 241 Huaihai Road (W), Shanghai 200030, China. Email: h_zhao28@163.com.

Background: The trachea surgery is generally performed due to stenosis, traumatic injury, foreign body and tumors with higher mortality and morbidity rates. Airway difficulties, hypoxia, stridor, cough, hemoptysis are common conditions in these patients.

Methods: We analyzed the medical records of trachea surgery patients treated at Shanghai Chest Hospital from 1961 to February 2019. Surgical treatment of various tracheal diseases was conducted in 933 cases, including 565 males and 368 females with mean age of 41 (range, 3-76) years. The number of tracheal annular resection and anastomosis is 592, other surgery procedures include 98 carina resection and reconstruction, 53 open window operation, 60 sleeve pneumonectomy, 30 laryngectomy and laryngotracheal reconstruction, 35 carina reconstruction, 28 cardiopulmonary bypass assisted tracheal resection, and 37 other cases in this group. For upper tracheal lesion (cross neck and low collar), the classic incision is selected at $1 \mathrm{~cm}$ above the clavicle head, and the length depends on the required exposure range above. For midtracheal lesion, transverse cervical incision combined with superior mediastinal incision (t-type incision) is conducted. The sternal incision is just above the sternal angle; total sternotomy is generally unnecessary and does not contribute to surgical field exposure. For lower trachea and carina lesions, right posterolateral incision (s-shaped incision, cut the 4th or 5 th rib) provide good exposure (including left main branch). Trachea surgery in complex high-risk situations, minimally invasive cardiopulmonary bypass (a-v) and extracorporeal membrane oxygenation (ECMO) technical assistance $(\mathrm{v}-\mathrm{v})$ can provide the opportunity to operate. The extracorporeal circulation was stopped after tracheal resection and intraoperative intubation. The in vitro time was about $15 \mathrm{~min}$. Thoracoscopic thoracic tracheal surgery and non-endotracheal intubation of the cervical trachea is also conducted.

Results: Three hundred and forty-five non-neoplastic cases includes 15 congenital trachea malformation, 171 traumatic stenosis, 77 iatrogenic stenosis, 75 trachea fistula and 7 tracheal tuberculosis. Of the 515 malignant tumors, 467 were primary and 48 were secondary malignant tumors, includes 198 adenoid cystic carcinoma, 167 trachea squamous cell carcinoma, 37 adenocarcinoma, 10 carcinoid, 12 small cell carcinoma, 6 adenosquamous carcinoma, 1 low-differentiated carcinoma, 1 pale cell carcinoma, 41 thyroid cancer affects the trachea, 24 mucoepidermoid carcinoma, 6 bronchitis fibroblastoma, 7 esophageal cancer affects the trachea, 2 trachea lymphoma, 1 large cell neuroendocrine carcinoma, 2 peripheral lymphoepithelioma-like carcinoma. The 55 benign tumors include 12 leiomyoma, 6 fibroids, 4 angiomas, 3 hamartoma, 3 cyst, 14 peripheral schwannoma, 3 plasma cell tumors, 1 basal lipoma, 4 fibrohistiocytoma and 5 chondroma. No intraoperative death occurred in 933 cases and 3 cases died during perioperative period. Two cases of allogeneic tracheal transplantation, one case was reexcised 2 weeks after surgery due to graft softening. One patient survived 6.5 years after surgery, but died of metastasis due to tumor recurrence. Postoperative complications include 22 postoperative anastomotic opening, 25 recurrent laryngeal nerve injury, 30 anastomotic restenosis.

Conclusions: Tracheal resection and reconstruction in the Shanghai Chest Hospital experience provide safe and useful methods for trachea patients. For complex high-risk trachea surgery, minimally invasive cardiopulmonary bypass and ECMO are useful.

Keywords: Tracheal resection; reconstruction; Shanghai Chest Hospital experience

doi: $10.21037 /$ shc.2019.AB007

Cite this abstract as: Zhao H, Yao F, Zhao Y, Gu HY. Modalities of tracheal resection and reconstructionthe Shanghai Chest Hospital experience. Shanghai Chest 2019;3:AB007. 